\title{
Lupus Anticoagulant Disorder
}

National Cancer Institute

\section{Source}

National Cancer Institute. Lupus Anticoagulant Disorder. NCI Thesaurus. Code C116994.

A hypercoaguable state that results from the presence of the immunoglobulin known as Lupus Anticoagulant. The antibody interacts with cell membrane phospholipids, causing increased aggregation and adhesion of platelets, which causes increased clot formation. Though the majority of patients who test positive for lupus anticoagulant do not have lupus, those individuals afflicted with lupus have a higher probability of developing the antibody. 